\title{
Modélisation du comportement au feu d'un composite par calcul de pyrolyse : approche combinée expérience-simulation à petite échelle
}

\author{
Damien Marquis ${ }^{1,2, a}$, Lucas Bustamante Valencia $^{1}{ }^{1}$ Éric Guillaume ${ }^{1}$, \\ Michel Pavageau ${ }^{2}$ et Carine Chivas ${ }^{1}$ \\ 1 Laboratoire National de métrologie et d'Essais (LNE), Direction des Essais (DE 522), Département comportement au feu et \\ sécurité incendie, 29 avenue Roger Hennequin, 78197 Trappes Cedex, France \\ 2 École des Mines de Nantes (EMN), Département Systèmes Énergétiques et Environnement, UMR CNRS 6144 (GEPEA), \\ 4 rue Alfred Kastler, BP 20722, 44307 Nantes, France
}

Reçu le 20 April 2009

\begin{abstract}
Résumé - Le travail présenté dans ce document s'inscrit dans le cadre d'un projet de recherche français de 3 ans dédié à l'utilisation de matériaux composites dans la construction navale. Dans ce projet, il est prévu d'utiliser le code CFD FDS-5 avec un modèle de pyrolyse pour tenir compte de la contribution de cloisons en matériau composite sur le développement du feu, dans le cadre de la simulation de scénarios incendies à bord d'un navire. Pour garantir la qualité de ces simulations, une méthode de validation progressive des modèles utilisés a été suivie. Cette méthode est basée sur une approche combinée expérience-simulation à échelle croissante. Pour illustrer la démarche, on présente ici les résultats d'une étude expérimentale du comportement au feu d'un échantillon de matériau composite, sur un banc d'essais à petite échelle, puis la simulation des essais correspondants.
\end{abstract}

Mots clés : Naval / feu / composites / simulations numériques / pyrolyse / échelle / expérimental

\begin{abstract}
Small scale validation of pyrolysis-model based CFD simulations of the fire behaviour of a laminate composite. The work presented in this paper comes within the framework of a 3 -year French collaborative research project dedicated to the use of composite materials in shipbuilding. In this project, it is planned to use the CFD code FDS5 with a pyrolysis model to better account for the behaviour of composite walls in the simulation of fires onboard a ship. Prior to the simulation of complete fire scenarios, numerical simulations of actual experiments performed on composite material samples of increasing size were performed for validation and evaluation purposes. To illustrate this approach, we present the results of an experimental study of the fire behaviour of a composite material at small scale, then the simulation of these tests for validation.
\end{abstract}

Key words: Ship / fire / composite / computations / pyrolysis / scaling / experiments

\section{Introduction}

Le travail présenté dans cet article s'inscrit dans le cadre du projet multi-partenaires MP08 intitulé « Comportement au feu des matériaux composites $»$. Ce projet, labellisé par le pôle de compétitivité EMC2 (Ensembles Métalliques et Composites Complexes) de la Région Pays de la Loire, rassemble des chantiers navals civils et militaires (DCNS, STX Europe et Bénéteau)

\footnotetext{
${ }^{a}$ Auteur pour correspondance : damien.marquis@lne.fr
}

voisins géographiquement autour d'une problématique commune, la tenue au feu des composites. L'objectif est de développer une démarche innovante permettant l'utilisation de ce type de matériaux, souvent inflammables, dans des domaines nouveaux ou jusqu'ici difficilement accessibles du fait des règles incendie en vigueur [1].

Les matériaux composites utilisés à des fins de construction, lorsqu'ils sont combustibles, constituent en cas d'incendie un terme source potentiellement important dont il faut pouvoir prévoir le comportement et la contribution à la propagation du feu. L'évaluation de l'énergie 


\section{Nomenclature}

\begin{tabular}{|c|c|}
\hline \multicolumn{2}{|c|}{ Lettres romaines } \\
\hline$A$ : & Facteur pré-exponentiel, $\mathrm{s}^{-1}$ \\
\hline$c:$ & 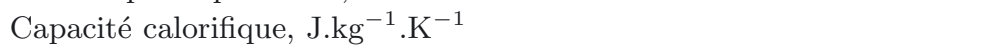 \\
\hline$E:$ & Énergie d'activation, $\mathrm{J} \mathrm{kg}^{-1}$ \\
\hline$\Delta H_{r, i}:$ & Enthalpie de réaction, ${\mathrm{J} . \mathrm{kg}^{-1}}^{-1}$ \\
\hline$k:$ & Conductivité thermique, $\mathrm{W} \cdot \mathrm{m}^{-1} \cdot \mathrm{K}^{-1}$ \\
\hline$n:$ & Ordre de la réaction, sans dimension \\
\hline$m:$ & Masse, $\mathrm{kg}$ \\
\hline$\dot{m}:$ & Vitesse de perte de masse totale, $\mathrm{s}^{-1}$ \\
\hline$\dot{q}:$ & Flux, $W$ \\
\hline$R:$ & Constante des gaz parfaits $\left(8,314472 \mathrm{~m}^{2} \cdot \mathrm{kg} \cdot \mathrm{s}^{-2} \cdot \mathrm{K}^{-1} \cdot \mathrm{mol}^{-1}\right)$ \\
\hline$t:$ & Temps, s \\
\hline$T:$ & Température, ${ }^{\circ} \mathrm{C}$ \\
\hline$Y:$ & Fraction massique, sans dimension \\
\hline \multicolumn{2}{|c|}{ Lettres greques } \\
\hline$\rho:$ & Masse volumique, $\mathrm{kg} \cdot \mathrm{m}^{-3}$ \\
\hline$\dot{\omega}:$ & Vitesses de réaction, $\mathrm{s}^{-1}$ \\
\hline$\phi:$ & Paramètre d'optimisation, sans dimension \\
\hline$v:$ & Coefficient stœchiométrique, sans dimension \\
\hline \multicolumn{2}{|l|}{ Indices } \\
\hline$i:$ & $i^{\text {ème }}$ réaction \\
\hline o : & Initial \\
\hline rad : & Radiatif \\
\hline $\mathrm{O}_{2}:$ & Dioxygène \\
\hline \multicolumn{2}{|c|}{ Exposants } \\
\hline$\cdot:$ & Par unité de temps \\
\hline$\square$ : & Par unité de volume \\
\hline * : & Normalisé \\
\hline \multicolumn{2}{|c|}{ Abréviations } \\
\hline $\mathrm{AG}:$ & Algorithme génétique \\
\hline ATG : & Analyse thermogravimétrique \\
\hline $\mathrm{CC}:$ & Cône calorimètre \\
\hline CFD : & Computional fluid dynamics \\
\hline FDS : & Fire dynamics simulator \\
\hline HRR : & Taux de dégagement de chaleur ( Heat release rate) W.m ${ }^{-2}$ \\
\hline MLR : & Vitesse de perte de masse (Mass loss rate), kg.s ${ }^{-1}$ \\
\hline NMLR : & Vitesse de perte de masse normalisée (Normalized mass loss rate), $\mathrm{s}^{-1}$ \\
\hline
\end{tabular}

apportée par la décomposition de ces matériaux, en cas de feu, est en effet essentielle pour prévoir la réaction et la résistance des structures, bien les dimensionner, et in fine assurer la sécurité des passagers en cas d'incident.

Ce travail d'analyse préalable, qui relève de l'ingénierie de la sécurité incendie [2], ne peut reposer sur des essais grandeur réelle que de manière très limitée. La démarche s'appuie donc largement sur l'emploi d'outils de calcul numérique, étant attendu de ces outils qu'ils permettent une prédiction réaliste de l'enchaînement des événements pouvant survenir en cas d'incendie.

Le code de calcul utilisé dans notre travail est capable de modéliser le développement d'un feu tout en tenant compte, à partir d'un modèle de pyrolyse, du comportement au feu de cloisons composites. Cette capacité permet a priori une reproduction plus fidèle du développement d'un incendie parce qu'elle tient compte dynamiquement de la décomposition des matériaux en présence, de leur contribution à la propagation des flammes, des dégagements de chaleur qui accompagnent la décomposition thermique des matériaux avec l'évolution des conditions ambiantes. La démarche la plus couramment utilisée, qui consiste à simuler un incendie à partir d'une courbe le taux de dégagement de chaleur imposée, ne permet pas de tenir compte des phénomènes énoncés ci-dessus. 


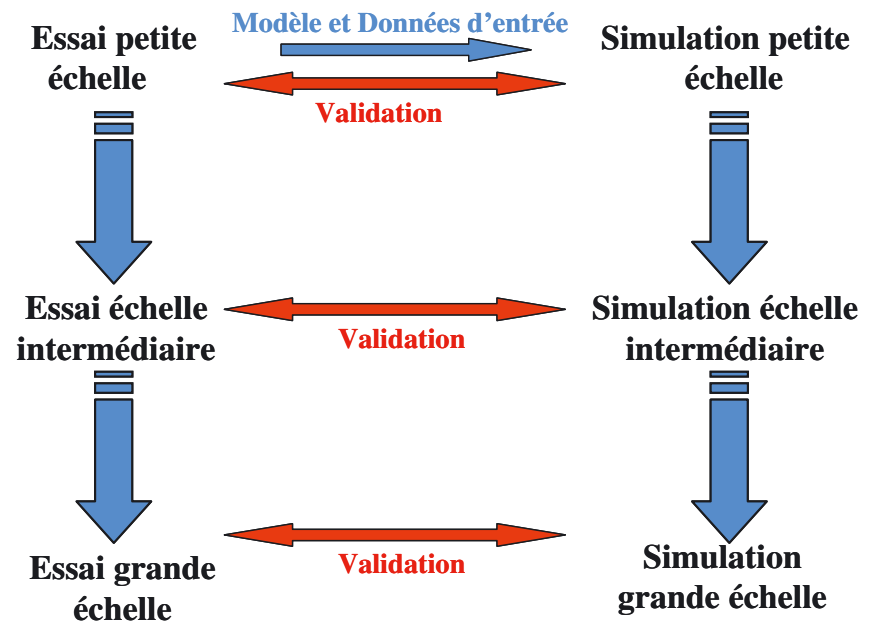

Fig. 1. Validation pas à pas par comparaison expériencesimulation à échelle croissante.

En contrepartie, une connaissance plus approfondie des propriétés thermophysiques des matériaux dont la combustion est simulée est nécessaire. Les cinétiques de combustion (produits, taux de réaction, ordre des modèles de décomposition, etc.) doivent être déterminées et précisées pour le calcul. Il est aussi nécessaire de s'assurer que les calculs reproduisent correctement le comportement de produits (panneaux, montants, ... ) constitués de ces matériaux quelles que soient la géométrie, l'orientation et la taille de ces produits.

Une approche aussi « raffinée » est-elle nécessaire? Les modèles de pyrolyse utilisés, déduits d'expériences sur des échantillons de taille réduite (de quelques millimètres à quelques centimètres) permettent-ils effectivement de reproduire, par le calcul, les expériences qui ont conduit à leur établissement? Sont-ils adaptés au calcul d'incendies sur des espaces de plusieurs dizaines de mètres correspondant à des domaines de calcul dont les mailles ont des dimensions de l'ordre de quelques centimètres?

Ainsi, une méthode de «validation » progressive basée sur une approche combinée expérience-simulation à échelle croissante [3] a été mise en œuvre pour mieux appréhender la qualité in fine de simulations numériques de scénarios incendies complets. La démarche, schématisée figure 1, comporte trois étapes :

- une phase expérimentale à échelle croissante permettant la caractérisation du comportement des matériaux étudiés dans différentes situations,

- une phase numérique consistant en la simulation de ces expériences,

- une phase de comparaison et de validation.

A chaque échelle d'observation, les résultats de la simulation numérique sont comparés à ceux de l'expérience au moyen de l'analyse fonctionnelle d'Hilbert décrite dans la norme ISO 16730 [4]. Chaque fois que c'est possible, cette comparaison porte d'une part sur des variables globales tels le taux de dégagement de chaleur ou la vitesse de perte de masse de l'éprouvette test, et d'autre part sur des variables locales comme des températures ou des vitesses locales de propagation de flamme.

Les écarts éventuels entre les résultats expérimentaux et numériques sont analysés. Les paramètres du modèle sont ajustés si nécessaire. L'idée de cette méthodologie est de converger progressivement vers un modèle adapté à la simulation de feux à échelle réelle.

Le présent document est restreint à l'étude du comportement au feu d'un matériau composite, à petite échelle uniquement.

\section{Modèle de pyrolyse}

\subsection{Matériau étudié}

Le matériau étudié est un matériau composite stratifié constitué d'une résine polyester et de fibres de verre de $4 \mathrm{~mm}$ d'épaisseur. Une caractérisation physico-chimique complète de ce matériau, éléments constitutifs séparés puis ensemble, a été réalisée au LNE au moyen de bancs d'essais et de méthodes de référence. Les informations correspondantes étant pour l'instant confidentielles, elles ne peuvent pas être données ici.

\subsection{Les bases d'un modèle de pyrolyse}

La décomposition thermique d'un matériau composite apparemment aussi «simple»que celui que nous avons étudié est en fait un processus complexe qui met en jeu de nombreux constituants chimiques et différents mécanismes. Il peut toutefois être commode dans un premier temps de décrire ce processus à partir de la connaissance des processus de décomposition thermique des éléments constitutifs du composite considéré pris séparément. Cette philosophie simplificatrice, qui ne prend pas en compte explicitement un certain nombre de couplages, est assez répandue.

La démarche requiert donc la définition préalable d'un schéma réactionnel et d'un modèle cinétique pour chaque élément constituant le composite. Ces schémas réactionnels sont généralement issus d'analyses thermogravimétriques $[5,6]$ et complétés par des observations effectuées au cône calorimètre. Dans notre cas, seule la résine a été soumise à une analyse thermo-gravimétrique. En effet, il n'y pas lieu d'introduire de modèle de décomposition pour les fibres de verre, elles sont incombustibles. Mais, l'influence de ces dernières sur le comportement au feu du stratifié a été étudiée au cône calorimètre.

La cinétique de décomposition de la résine polyester dépend fortement des conditions d'essais [7]. De ce fait, des essais ATG ont été réalisés à différentes vitesses d'échauffement et sous différents gaz vecteurs oxydants et non-oxydants. Les résultats de ces essais ont permis d'établir un schéma réactionnel pour la résine polyester. Le tableau 1 résume les quatre étapes de la décomposition thermique de la résine. 
Tableau 1. Schéma réactionnel d'une résine polyester déduit d'une analyse ATG.

\begin{tabular}{ccccc}
\hline & Type de & Reactif & & Produit \\
& reaction & & & \\
\hline 1 & Pyrolyse & Resine & $\rightarrow$ & $v_{1} \cdot$ charbon $+\left(1-v_{1}\right) \cdot$ gaz \\
2 & Oxydation & Resine $+\mathrm{O}_{2}$ & $\rightarrow$ & $v_{2} \cdot \beta$ resine $+\left(1-v_{2}\right) \cdot g a z$ \\
3 & Oxydation & $\beta$ resine $+\mathrm{O}_{2}$ & $\rightarrow$ & $v_{3} \cdot$ charbon $+\left(1-v_{3}\right) \cdot g a z$ \\
4 & Oxydation & charbon $+\mathrm{O}_{2}$ & $\rightarrow$ & $v_{4} \cdot$ residu $+\left(1-v_{4}\right) \cdot$ gaz \\
\hline
\end{tabular}

La vitesse de réaction $\dot{\omega}_{i}$ de chacune des quatre réactions du tableau 1 est décrite par une loi de type Arrhenius (Eq. (1)) où $i$ est le numéro de la réaction considérée :

$$
\dot{\omega}_{i}(t)=A_{i} \mathrm{e}^{\frac{-E_{i}}{R T}}\left(\frac{m_{i}(t)}{m_{o}}\right)^{n_{i}} Y_{\mathrm{O}_{2}}^{\delta}
$$

$m_{i}$ la masse du réactif de la réaction $i, m_{o}$ la masse initiale du matériau et $Y_{\mathrm{O}_{2}}=0,23$ la fraction massique d'oxygène. Pour une réaction de décomposition thermique par pyrolyse l'exposant $\delta$ est nul. Il vaut 1 pour une réaction de décomposition thermo-oxydative.

La vitesse de perte de masse totale normalisée par la masse initiale de la résine $\dot{m}^{*}(t)$ (ou NMLR) est décrite par l'équation (2)

$$
\dot{m}^{*}(t)=\sum_{i=1}^{N} \dot{m}_{i}^{*}(t)=\sum_{i=1}^{N}\left(v_{i}-1\right) \dot{\omega}_{i}(t)
$$

$N$ est le nombre total de réactions du modèle et $v_{i}$ le coefficient stœchiométrique de la réaction $i$.

Les conditions initiales sont données par les égalités (3) :

$$
\left\{\begin{array}{l}
m_{\text {resine }}(t=0)=1 ; m_{\text {resine }}(t=0)=0 \\
m_{\text {charbon }}(t=0)=0 ; m_{\text {residu }}(t=0)=0 \\
T(t=0)=393 \mathrm{~K})
\end{array}\right.
$$

L'équation (2) permet de calculer la vitesse de perte de masse normalisée $\dot{m}^{*}(t)$ en fonction de la température via l'équation (1).

\subsection{Technique de détermination des paramètres cinétiques du modèle}

Les paramètres cinétiques de l'équation (1) sont estimés à partir d'une méthode d'optimisation itérative conduite par un algorithme génétique (AG) [9-11], outil qui s'est révélé adapté à la résolution de ce genre de problème fortement non linéaire [12].

La technique consiste à minimiser l'écart relatif entre la courbe $\dot{m}^{*}(t)$ expérimentale et la courbe $\dot{m}^{*}(t)$ issue d'un modèle de la forme de l'équation (1) et d'un jeu de paramètres cinétiques $\left(E_{i}, A_{i}, n_{i}\right.$ et $\left.v_{i}\right)$ en cherchant à optimiser le paramètre $\phi$ défini par :

$$
\phi=\cos [\angle(\vec{x}, \vec{y})] \cdot\left[\frac{\|\vec{x}-\vec{y}\|}{\|\vec{x}\|}\right]^{-1}
$$

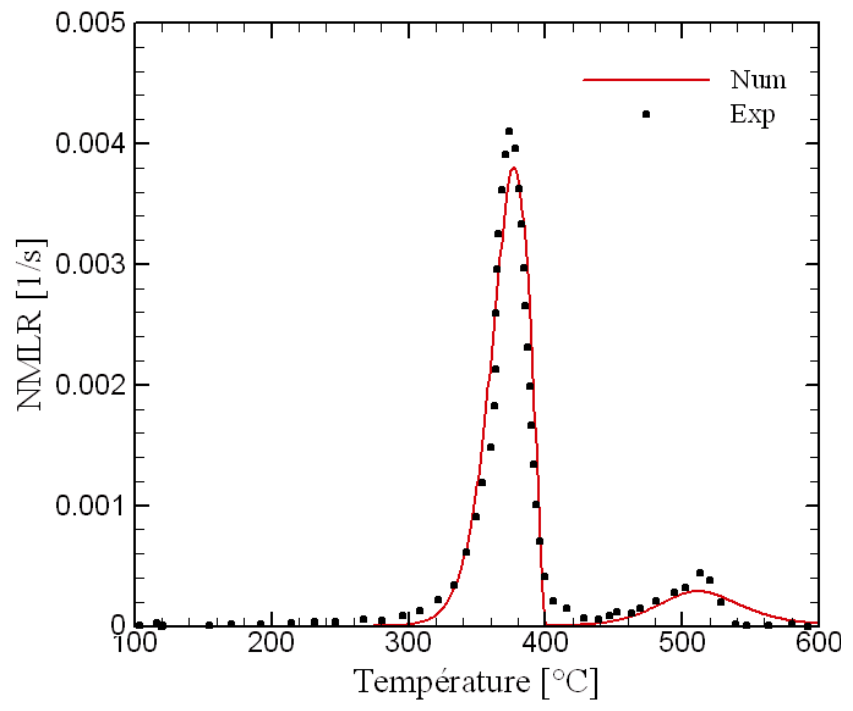

Fig. 2. Comparaison entre la solution calculée par l'AG et les valeurs expérimentales pour une vitesse de chauffe de $10 \mathrm{~K} \mathrm{~min}^{-1}$ en milieu oxydant.

Dans l'équation (4), $\vec{x}$ est un vecteur formé des valeurs expérimentales $\dot{m}^{*}(t)$ et $\vec{y}$ un vecteur formé des valeurs de $\dot{m}^{*}(t)$ issues du modèle. Le paramètre $\phi$ fait appel à un produit scalaire noté $\langle\vec{x}, \vec{y}\rangle$ et à la norme associée qui sont donnés dans la norme ISO 16730 [4] laquelle définit en fait 4 produits scalaires : euclidien, d'Hellinger, sécante et hybride.

Le cosinus de l'angle entre les vecteurs $\vec{x}$ et $\vec{y}$ défini par l'équation (5) ci-dessous est représentatif de la colinéarité des vecteurs, c'est-à-dire de la concordance morphologique des courbes expérimentales et numériques. Plus sa valeur est proche de 1 , meilleure est la concordance morphologique entre les courbes représentées par les vecteurs $\vec{x}$ et $\vec{y}$.

$$
\cos [\angle(\vec{x}, \vec{y})]=\frac{\langle\vec{x}, \vec{y}\rangle}{\|\vec{x}\|\|\vec{y}\|}
$$

L'AG calcule $\phi$ jusqu'à ce que le NMLR calculé converge vers le NMLR expérimental. Les paramètres cinétiques optimisés seront ensuite utilisés comme jeu de données pour simuler le comportement au feu de la résine polyester. Inutile de préciser que le choix de la fonction d'optimisation $\phi$ influe sur les valeurs des paramètres du modèle [10].

\subsection{Application et résultat}

La figure 2 présente les NMLR expérimentaux et calculés pour la résine seule, en milieu oxydant. Le modèle retenu (Tab. 1) reproduit de manière satisfaisante les principales tendances observées expérimentalement pour une vitesse d'échauffement de la résine de $10 \mathrm{~K} . \mathrm{min}^{-1}$. Ce modèle de décomposition de la résine seule (schéma réactionnel et jeu de paramètres cinétiques) a été validé pour d'autres vitesses d'échauffement. Il a été utilisé pour 


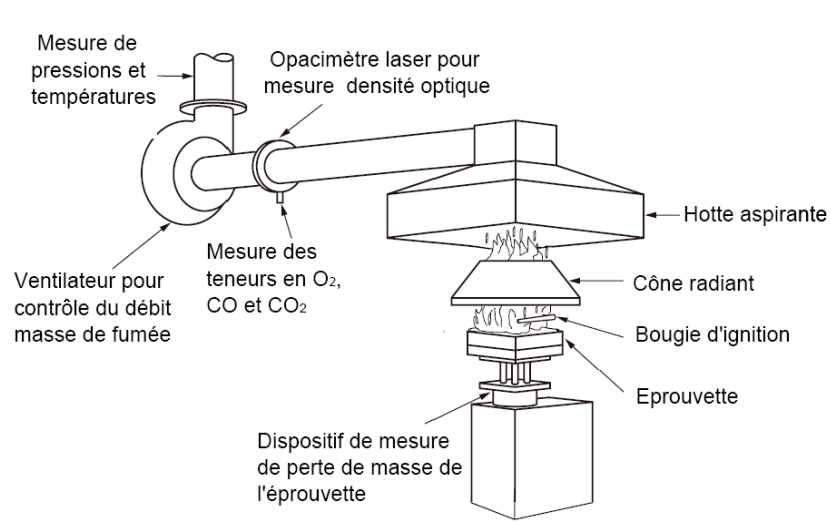

Fig. 3. Cône calorimètre.

la simulation numérique du comportement au feu du composite décrite dans la section suivante.

\section{Expériences et simulations au cône calorimètre}

\subsection{L'expérience : le cône calorimètre}

Le cône calorimètre est un dispositif de mesure permettant l'évaluation du HRR d'un matériau lors de sa combustion. Le principe de la mesure repose sur la calorimétrie par déplétion d'oxygène, la quantité d'énergie libérée par la combustion d'un échantillon du matériau étant donc déterminée par la mesure de la consommation d'oxygène durant la combustion. L'éprouvette, parallélépipédique, de $100 \mathrm{~mm} \times 100 \mathrm{~mm}$ et d'épaisseur l'épaisseur de l'échantillon à tester est placée dans un porte-éprouvette normalisé (face arrière de l'échantillon testé isolée). Celui-ci est positionné sur une balance qui permet de suivre en continu l'évolution de la perte de masse. L'échantillon est exposé au flux d'un radiateur électrique conformément aux exigences de la norme ISO $5660-1$ (voir Fig. 3).

\subsection{La simulation numérique : le code FDS 5}

Fire Dynamics Simulator version 5.3 est un code CFD couplé à des modèles de combustion et de pyrolyse. FDS utilise comme modèle hydrodynamique une approximation des équations de Navier-Stokes appropriée à la simulation des écoulements à bas nombre de Mach. La turbulence est traitée par une approche LES à constante de Smagorinsky fixe dans tout le domaine de calcul. Pour le matériau, l'équation du bilan d'énergie est écrite à l'aide d'un modèle de transfert conductif monodimensionnel (Eq. (6)) :

$$
\bar{\rho} \bar{c} \frac{\partial T}{\partial t}=\frac{\partial}{\partial x}\left(k \frac{\partial T}{\partial x}\right)+\sum_{i=1}^{N} \Delta H_{r, i} \frac{\partial \rho_{i}}{\partial t}+\dot{q}_{r a d}^{\prime \prime \prime}
$$

L'indice $i$ renvoie à chaque constituant du matériau.

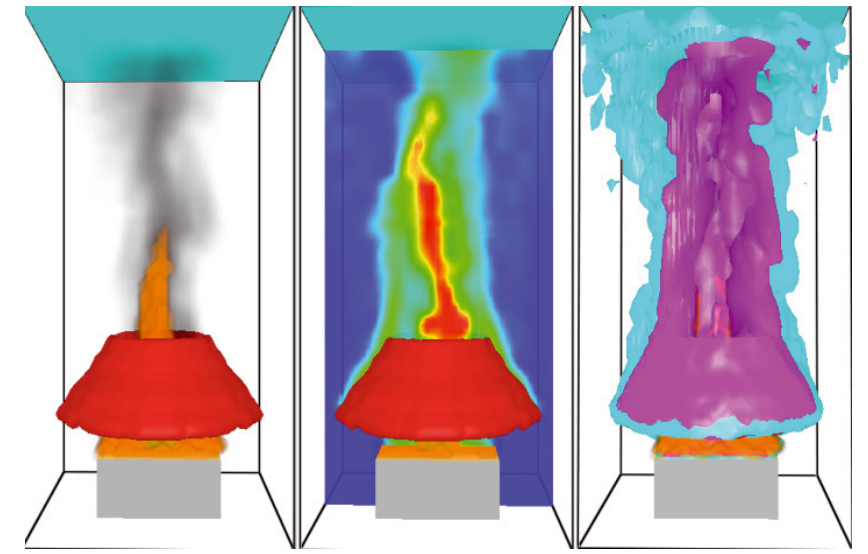

Fig. 4. Cône calorimètre virtuel, représentation des flammes et des fumées, du champ de température, et des isotempératures.

\subsection{Comparaison entre l'expérience et la simulation numérique}

Une simulation du cône calorimètre a été réalisée en respectant les dimensions réelles du banc d'essai expérimental (Fig. 4). Le modèle de décomposition de la résine défini précédemment a été intégré au code de calcul.

Il s'agit maintenant de vérifier que l'expérience virtuelle du cône calorimètre permet bien de reproduire le comportement au feu de la résine seule puis du composite étudié. La comparaison entre expérience et simulation porte ici simplement sur le bilan global de masse. Elle n'a pas pu être complétée par la comparaison de grandeurs locales car l'expérience du cône calorimètre ne permet pas la mesure de températures locales ni de vitesses de convection au dessus ou au voisinage de l'échantillon. Les simulations numériques sont comparées aux résultats expérimentaux en utilisant l'analyse fonctionnelle d'Hilbert comme précédemment.

\subsubsection{Résine polyester seule}

La figure 5 compare le MLR et le HRR de la résine seule obtenus numériquement et expérimentalement pour un flux incident de $50 \mathrm{~kW} \cdot \mathrm{m}^{-2}$.

Après l'ignition, le HRR augmente rapidement jusqu'à atteindre un maximum en un peu moins de $200 \mathrm{~s}$. À ce stade, plus de $70 \%$ de la partie organique de la résine est dégradée. Après ce pic, le HRR diminue rapidement. Cette décroissance est liée à un réarrangement moléculaire du polymère favorisant la formation d'une structure de type carbonée (charbon) thermostable. Après 200 s, une réaction thermo-oxydative est observée sur ce charbon produisant la dégradation totale de la partie organique.

Le modèle de décomposition permet une reproduction correcte du MLR et du HRR. Après l'inflammation, le taux de perte de masse numérique est légèrement supérieur au MLR expérimental. Cet écart peut être dû 

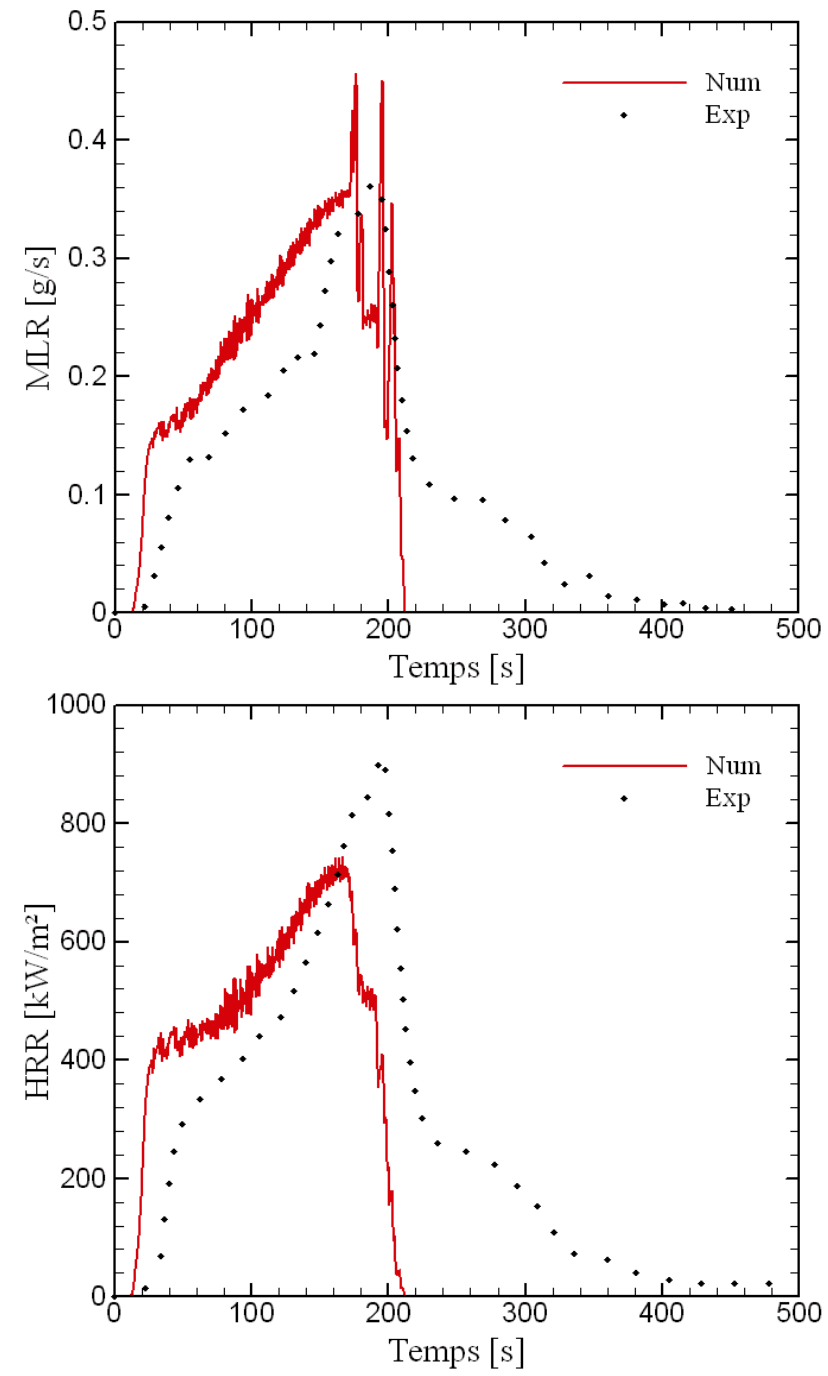

Fig. 5. Comparaison entre résultats numériques et expérimentaux pour la résine polyester seule pour un niveau d'irradiance de $50 \mathrm{~kW} \cdot \mathrm{m}^{-2}$.

à une sur-estimation par le modèle de pyrolyse du taux d'oxydation du charbon. En revanche, au niveau du pic, le code sous-évalue fortement l'énergie dégagée par la résine, tandis que le MLR numérique tend vers la mesure expérimentale. Cette différence de HRR indique que l'enthalpie de combustion effective utilisée dans le code est légèrement trop faible.

Le modèle utilisé ne permet pas de reproduire correctement la dernière réaction d'oxydation qui apparaît après $200 \mathrm{~s}$ d'exposition du matériau au flux de chaleur. De grosses instabilités numériques sont observées sur le MLR lors de cette phase de décomposition.

Afin de quantifier l'écart, les résultats de l'analyse fonctionnelle sur $220 \mathrm{~s}$ du bilan de masse de la résine polyester sont résumés dans le tableau 2. Dans le cas présent, la valeur du cosinus de l'angle de la norme hybride montre globalement un faible éloignement en forme (cosinus de l'angle proche de un). La norme euclidienne montre que l'écart relatif entre les vecteurs est de l'ordre de 21,6\%
Tableau 2. Analyse de comparaison sur la résine polyester seule.

\begin{tabular}{ccc}
\hline & Norme Euclidienne & Norme Hybride \\
\hline$\|\vec{x}\|$ & 2,054 & 0,207 \\
$\|\vec{y}\|$ & 2,498 & 0,253 \\
Ecart relatif $(\%)$ & $-21,6$ & $-22,1$ \\
$\operatorname{Cos} \theta$ & 0,965 & 0,959 \\
\hline
\end{tabular}

Tableau 3. Analyse de comparaison sur la résine polyester seule.

\begin{tabular}{ccc}
\hline & Norme Euclidienne & Norme Hybride \\
\hline$\|\vec{x}\|$ & 0,68 & 0,069 \\
$\|\vec{y}\|$ & 0,639 & 0,065 \\
Ecart relatif (\%) & 6 & 5 \\
$\operatorname{Cos} \theta$ & 0,92 & 0,908 \\
\hline
\end{tabular}

et que le déphasage est faible (cosinus de l'angle proche de un).

Le modèle de pyrolyse introduit dans FDS ne traduit donc pas tout à fait la réalité des réactions physico-chimiques. Toutefois, la validation reste satisfaisante devant les incertitudes expérimentales (supérieures à $10 \%$ ) et l'écart-type de répétabilité des résultats expérimentaux.

\subsubsection{Résine polyester et fibres de verre}

La figure 6 permet de comparer les comportements numériques et expérimentaux du composite stratifié (résine polyester avec fibres de verre) via le MLR et le HRR obtenus pour un flux incident de $50 \mathrm{~kW} . \mathrm{m}^{-2}$.

Concernant le HRR, les courbes expérimentales et numériques présentent une allure similaire bien que la simulation numérique prédise une inflammation plus rapide de l'éprouvette. Après le premier pic, le HRR diminue rapidement. Cette décroissance est liée au ralentissement de la décomposition du composite en raison d'un épaississement de la couche de charbon qui se forme à la surface du matériau. Les fibres de verre réduisent également le HRR et agissent comme une barrière thermique. Le second pic, beaucoup plus large que le premier, est observé uniquement lorsque la face arrière de l'éprouvette est isolée (les conditions limites en face arrière ont donc un effet important sur la pyrolyse d'un matériau thermiquement fin). Il est attribué à une augmentation du taux de gazéification au sein de la partie imbrûlée du substrat et à l'augmentation de la température au sein du stratifié [12].

Quantitativement, le MLR numérique est inférieur aux résultats expérimentaux. Cet écart est lié à la mauvaise reproduction de la réaction de décomposition du charbon de la résine polyester (Fig. 5).

Afin de quantifier l'écart, les résultats de l'analyse fonctionnelle du bilan de masse sur le composite sont résumés dans le tableau 3. Dans le cas présent, la norme hybride montre que les courbes sont très proches en 

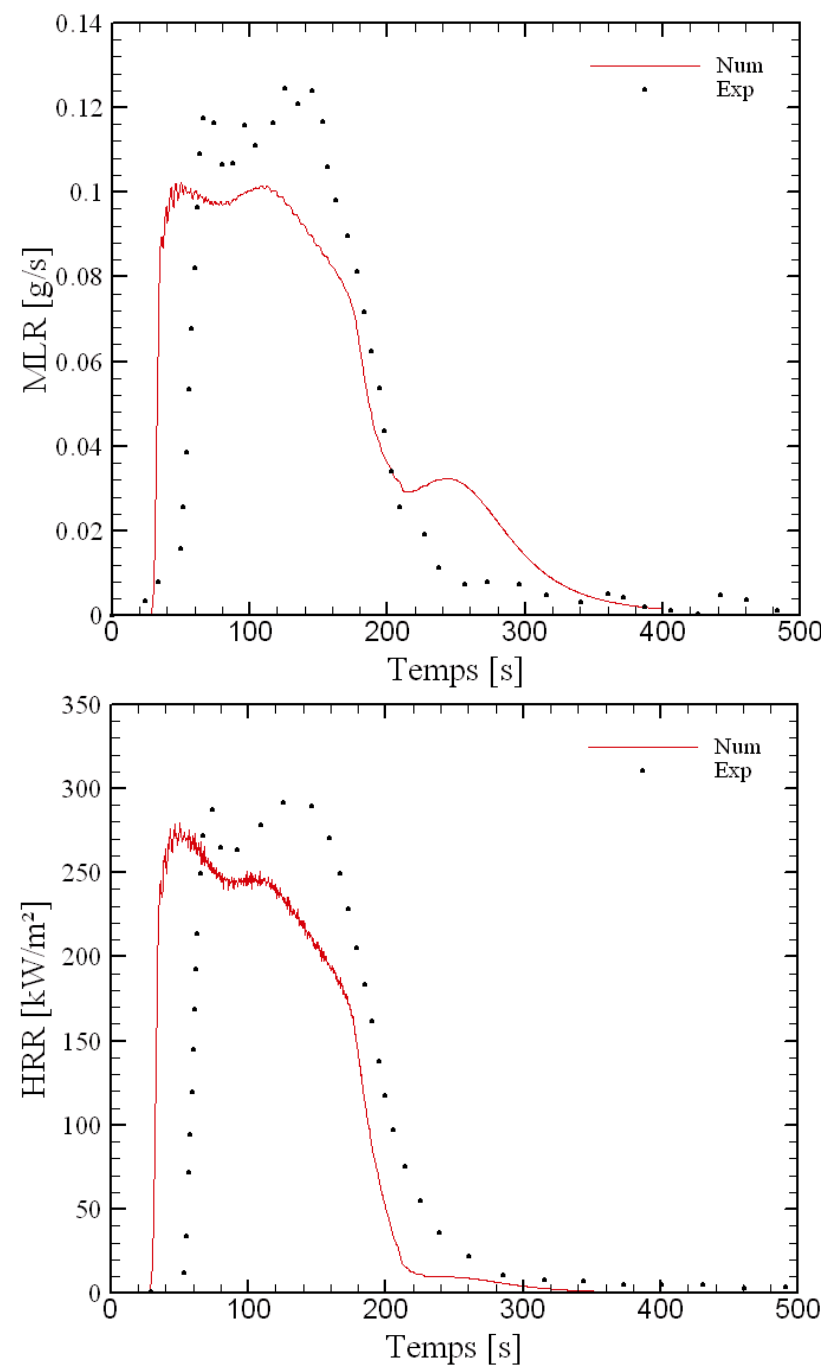

Fig. 6. Comparaison entre résultats numériques et expérimentaux du composite stratifié pour un niveau d'irradiance de $50 \mathrm{~kW} \cdot \mathrm{m}^{-2}$.

forme (cosinus de l'angle proche de l'unité). La norme euclidienne traduit un déphasage réduit et montre un désaccord entres les vecteurs de l'ordre de $6 \%$.

\subsubsection{Influence du maillage}

La taille des cellules du maillage est l'un des paramètres conditionnant une bonne simulation. En général, plus un maillage est fin, plus la simulation numérique est fidèle à l'expérimental. FDS utilise une précision de second ordre spatio-temporelle. De ce fait, l'augmentation du maillage par 2 engendre une division par 4 de l'erreur de discrétisation.

Les résultats d'une étude de sensibilité au maillage sur le comportement au feu du composite pour une densité de flux de $50 \mathrm{~kW} \cdot \mathrm{m}^{-2}$ sont présentés sur la figure 7 .

À cette échelle, l'augmentation de la taille des mailles conduit à un échauffement plus rapide du composite et, par conséquent, à une élévation de la vitesse de perte
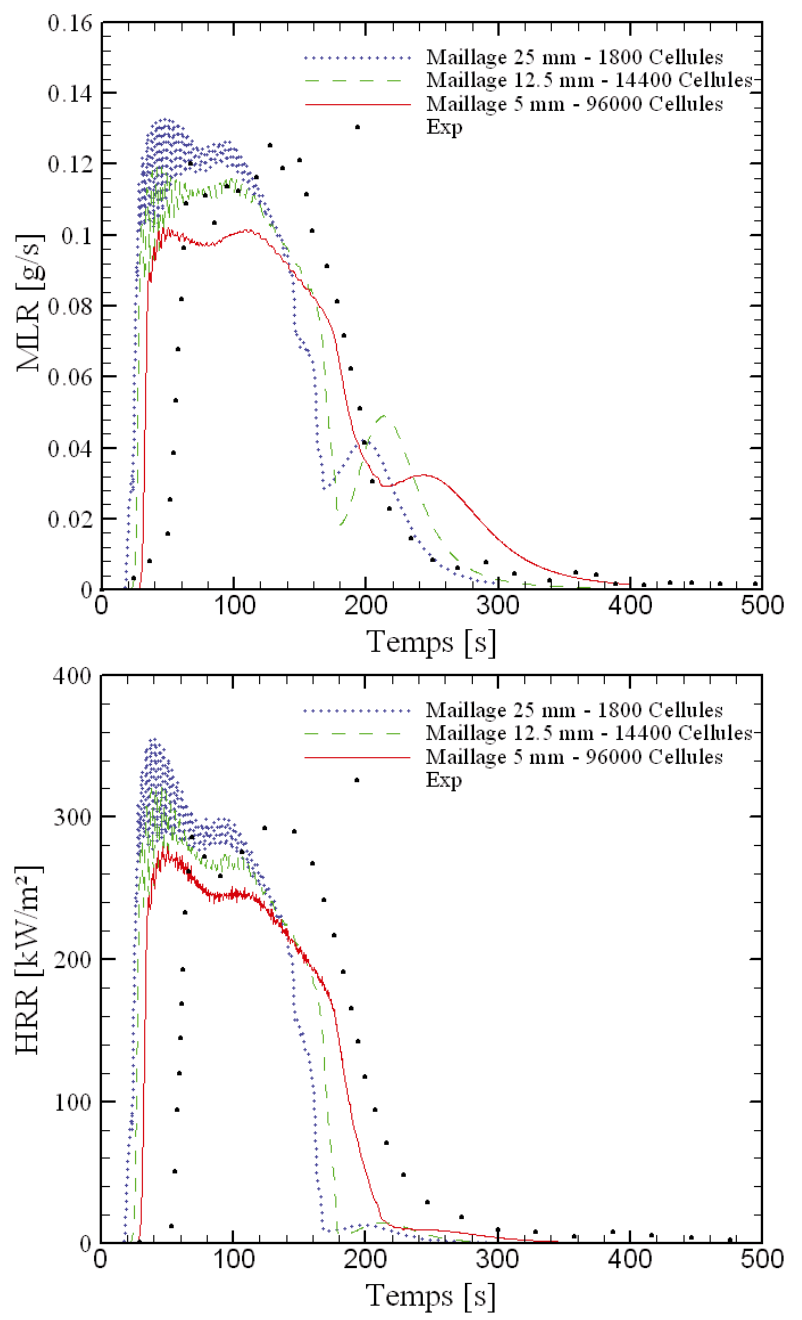

Fig. 7. Influence du maillage sur le comportement au feu du composite stratifié pour un flux incident de $50 \mathrm{~kW} . \mathrm{m}^{-2}$.

de masse. La durée de la combustion s'en trouve réduite tandis que l'énergie dégagée est amplifiée.

Par conséquent, l'augmentation du maillage va donc conduire à favoriser l'ignition et à sur-évaluer l'énergie dégagée par le matériau.

\subsubsection{Influence du flux incident}

Le code de calcul utilisé dans notre travail est capable de simuler le comportement au feu du matériau composite quel que soit le niveau d'irradiance. Des simulations numériques pour des densités de flux thermique de 35 , 50 et $75 \mathrm{~kW} \cdot \mathrm{m}^{-2}$ ont été réalisées avec le même jeu de paramètres en ce qui concerne le modèle de pyrolyse. La figure 8 présente les résultats obtenus.

Les résultats de la perte de masse sont très satisfaisants quel que soit le flux incident. À faible niveau d'irradiance, les résultats montrent que la simulation numérique accélére l'inflammation du stratifié. Le déphasage avec le délai d'ignition réel diminue fortement en fonction de l'augmentation du flux. De fait, le modèle 

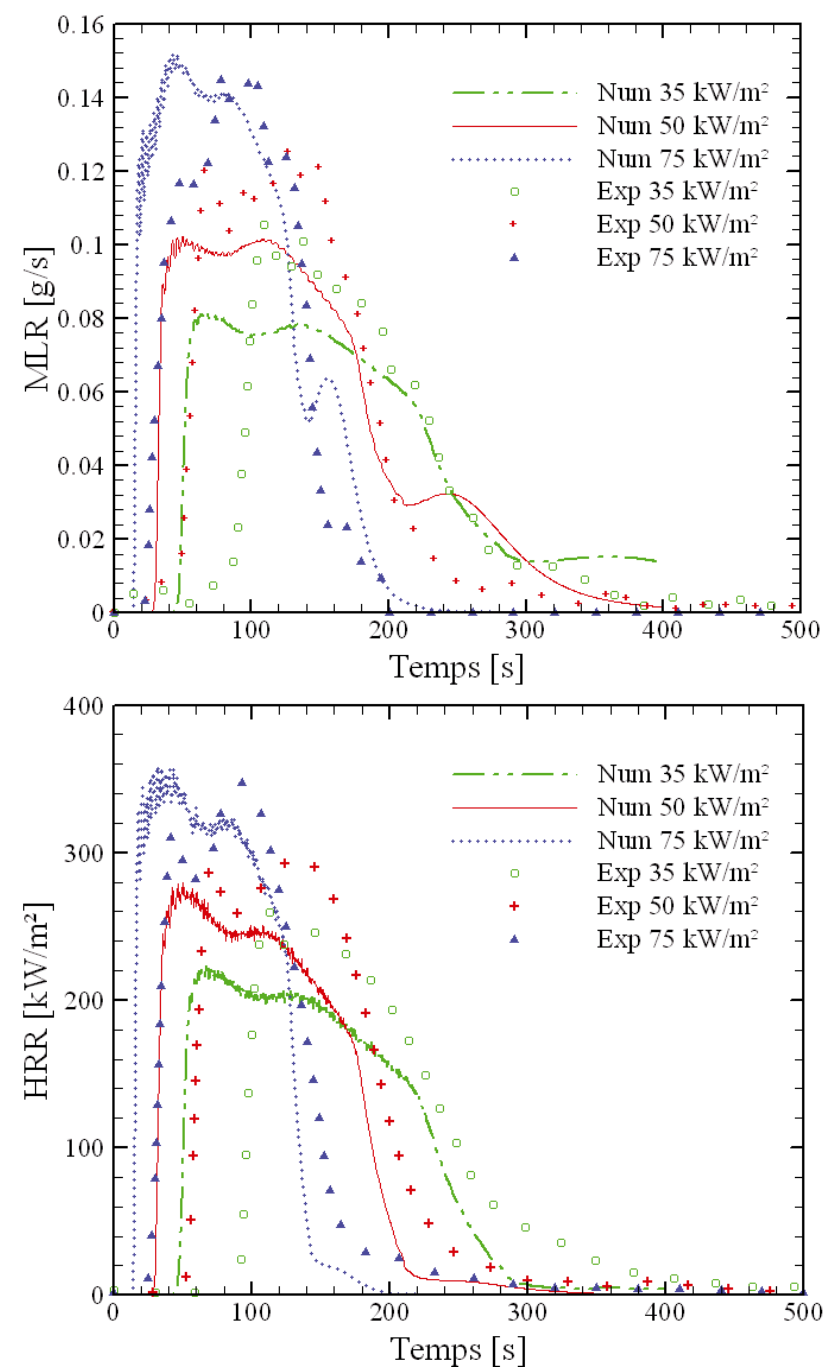

Fig. 8. Influence du niveau d'irradiance (35,50 et $\left.75 \mathrm{~kW} . \mathrm{m}^{-2}\right)$ sur la simulation du comportement au feu du composite stratifié.

sous-évalue l'énergie dégagée par le matériau ainsi que la perte de masse.

\subsubsection{Remarques concernant FDS}

Il est à noter que FDS utilise une équation de la chaleur mono-dimensionnelle. Cette simplification est justifiée dans FDS qui a été conçu pour simuler des incendies (de grandes dimensions) au cours desquels les parois en présence sont supposées être exposées de manière uniforme à un flux radiatif donné. Elle a pour effet un échauffement plus rapide du matériau qu'en réalité, car la chaleur ne peut pas diffuser latéralement. En outre, dans FDS, une molécule de gaz brûle instantanément pour peu que la concentration d'oxygène nécessaire soit présente (pas besoin d'énergie d'activation). Les flammes numériques ont donc tendance à se propager plus rapidement que les flammes réelles.
À l'échelle du cône calorimètre, les effets de bords sont également très importants. Les pertes de chaleur au travers des côtés non exposés de l'échantillon ne peuvent pas être simulées correctement. Les délais d'ignition numériques sont donc plus courts que les délais d'ignition réels.

\section{Conclusion}

Cet article présente d'une part, les résultats expérimentaux du comportement au feu d'une résine polyester et d'un composite stratifié (résine polyester avec fibres de verre), sur un banc d'essai à petite échelle (cône calorimètre) et d'autre part, les simulations numériques de ces essais à l'aide du code LES FDS 5 . Un modèle de pyrolyse du matériau considéré a en outre été utilisé.

Les simulations numériques ont permis de démontrer que le modèle de décomposition issu de l'analyse thermogravimétrique et le jeu de données expérimental permettaient une reproduction correcte du comportement au feu d'une résine polyester et d'un stratifié polyester-verre.

La comparaison effectuée est la première étape d'une approche multi-échelle qui doit permettre d'étudier pas à pas la capacité du modèle de pyrolyse à reproduire le comportement au feu du matériau étudié dans un grand nombre de scénarios incendie pour lesquels des essais à échelle réelle ne sont pas envisageables.

Les premiers résultats montrent qu'à l'échelle du cône calorimètre, le modèle de pyrolyse utilisé reproduit de manière satisfaisante les principales tendances observées expérimentalement en ce qui concerne la décomposition du matériau testé et ce quel que soit le niveau d'irradiance. Les simulations ont montré l'influence de la taille des mailles du domaine de calcul sur le calcul de la dégradation thermique du composite.

La prochaine étape de notre travail consistera à étudier expérimentalement et numériquement, selon la procédure décrite ici, le comportement au feu de ce matériau composite à plus grande échelle.

Remerciements. Les auteurs remercient le pôle de compétitivité EMC2, la région Pays de la Loire ainsi que la Direction Générale des Entreprises et la Délégation Générale de l'Armement pour leur soutien financier à la réalisation du projet MP08 cadre de ce travail.

\section{Références}

[1] J. Gutierrez, A. Breuillard, P. Corrignan, D. Marquis, C. Chivas, E. Guillaume, M. Pavageau, Use of fire safety engineering for the design of composites for shipbuilding, Proceedings of Comp. In Fire 5, Newcastle, Grande Bretagne, 2008

[2] IMO MSC/Circ.1002 Guidelines on Alternative Design and Arrangements for Fire Safety, 2001

[3] D. Marquis, M. Pavageau, E. Guillaume, C. Chivas, Multiscale validation of pyrolysis model based CFD Simulation of Fire growth on composite, Proceedings of the 11th international conference Fire \& Materials, Etats Unis, 2009, pp. 413-424 
[4] ISO 16730 : 2007 (E) : Fire safety engineeringAssessment, verification and validation of calculation methods

[5] C. Beyler, M. Hirschler, Thermal decomposition of polymers, SFPE Handbook of Fire Protection Engineering, 2nd Editon, 1995, pp. 99-199

[6] J. Conesa, A. Marcilla, J. Caballero, R. Font, Comments on the validity and utility of the different methods for kinetic analysis of thermogravimetric data. J. Anal. App. Pyr. 58-59 (2001) 617-633

[7] S. Vyazovkin, Thermal analysis, Anal. Chem. 80 (2008) 4301-4316

[8] G. Rein, C. Lautenberger, C. Fernandez Pello, J.L. Torero, D.L. Urban, Application of genetic algorithms and thermogravimetry to determine the kinetics of polyurethane foam in smoldering combustion, Comb. fl. 146 (2006) 95-108
[9] C. Lautenberger, G. Rein, C. Fernandez-Pello, The application of a genetic algorithm to estimate material properties for fire modeling from bench-scale fire test data. Fire Saf. J. 41 (2006) 204-214

[10] L.B. Valencia, T. Rogaume, E. Guillaume, New method for simuling the kinectic of toxic gases productions of upholstered furniture fire, Proceedings of the 11th international conference Fire \& Materials, Etats Unis, 2009, pp. 685-695

[11] C. Houck, J. Joines, J., A genetic algorithm for function optimization : a Matlab implementation, Report NCSUIE TR 9509. http://www.ise.ncsu.edu/

[12] J.-E. Brown, E. Braun, W.-H. Twilley, Cone calorimeter evaluation of the flammability of composite materials, Technical report NBSIR 88-3733, National bureau of standards, Center for fire research, 1988 